\title{
Selective separation of oil and water with mesh membranes by capillarity
}

\author{
Yuanlie Yu ${ }^{\text {a }}$, Hua Chen ${ }^{\text {b }}$, Yun Liu ${ }^{c}$, Vincent S. J. Craig ${ }^{\text {d, }}$, Zhiping Lai ${ }^{\text {a, }}$ \\ ${ }^{a}$ Advanced Membrane and Porous Materials Center, Division of Physical Science and Engineering, King Abdullah \\ University of Science and Technology, Thuwal 23955-6900, Saudi Arabia \\ ${ }^{\mathrm{b}}$ Centre for Advanced Microscopy, Australian National University, Canberra, ACT 0200, Australia \\ ${ }^{\mathrm{c}}$ Research School of Chemistry, Australian National University, Canberra, ACT 0200, Australia \\ ${ }^{\mathrm{d}}$ Department of Applied Mathematics, Research School of Physics and Engineering, Australian National University, \\ Canberra, ACT 0200, Australia
}

\begin{abstract}
The separation of oil and water from wastewater generated in the oil-production industries, as well as in frequent oil spillage events, is important in mitigating severe environmental and ecological damage. Additionally, a wide arrange of industrial processes require oils or fats to be removed from aqueous systems. The immiscibility of oil and water allows for the wettability of solid surfaces to be engineered to achieve the separation of oil and water through capillarity. Mesh membranes with extreme, selective wettability can efficiently remove oil or water from oil/water mixtures through a simple filtration process using gravity. A wide range of different types of mesh membranes have been successfully rendered with extreme wettability and applied to oil/water separation in the laboratory. These mesh materials have typically shown good durability, stability as well as reusability, which makes them promising candidates for an ever widening range of practical applications.
\end{abstract}

\footnotetext{
${ }^{*}$ Corresponding author: E-mail address: vince.craig@anu.edu.au, and zhiping.lai@,kaust.edu.sa
} 
Keywords: Mesh membranes; superhydrophobicity; superoleophobicity; oil/water separation; Capillarity

Contents

1. Introduction

1.1 Need for oil/water separation

1.2 Weak assembly of mesh membranes

1.3 Theory of superhydrophobicity and superhydrophilicity

1.4 Capillary basis for separation

2. Materials for coating mesh membranes for oil-water separation

2.1 Polymers

2.2 Boron nitride nanotubes

2.3 Metal oxides and hydroxides

2.4 Graphene oxide

2.5 Diamond-like carbon

2.6 Metals

2.7 Zeolites

3. Choice of membrane materials

4. Conclusions

Acknowledgments

References 


\section{Introduction}

\subsection{Need for oil/water separation}

The separation of oil and water from oil/water mixtures is an ancient challenge that is becoming more important [1-3]. Discharged oil can cause severe environmental and ecological damage directly and because the oil is flammable and can decompose to form other harmful chemicals, further polluting the natural environment and threatening aqueous habitats and affecting human health [2]. Furthermore, chemicals used in clean-up operations can cause environmental harm. Apart from these environmental and ecological issues, a wide range of industrial processes require the separation of oils or fats from aqueous solutions and the scarcity of water in many arid regions adds to the demand for efficient oil/water separation technologies [4]. Another important consideration in choosing the technology used to effect separation is the possible reuse of the recovered oil. Although traditional methods such as skimmers, centrifuges, coalesces, settling tanks, depth filters, magnetic separations, flotation technologies, and ignition of oil can remove oil from oily wastewater, these techniques are mainly useful for oil water mixtures and unstable emulsified oils, as their working mechanisms are sensitive to the oil droplet size and/or the density difference between water and oil [5-7]. Moreover, because they require long processing times or gas or chemical input, these conventional separation systems usually are expensive and require a large amount of space and energy $[5,6]$. More stringent environmental controls and the pressure of economic development have led to a strong desire to produce more efficient, cost-effective processes for oil and water separation [8].

Utilizing engineered wetting of solid surfaces to design an oil/water separation process based on capillary processes has been recognized as an effective approach [9-11]. For practical 
applications it is necessary to preferentially maintain one phase at the surface in addition to preventing fouling of the surfaces, therefore materials with extreme surface wettability of two types are employed. These are solids with surfaces that are superhydrophobic and superoleophilic or surfaces with the inverse wettability that are superoleophobic and superhydrophilic. Such surfaces when exposed to mixtures of oil and water preferentially attract one of the phases in order to reduce the overall interfacial energy of the system.

\subsection{Weak assembly}

From a molecular viewpoint the interfacial energy arises due to the loss of cohesion energy when a molecule is moved from the bulk to the interface. For liquid oil, we can approximate the cohesive energy of a molecule to the translational kinetic energy. Assuming half the cohesive interactions are lost for a molecule at the interface, the molecular energy shortfall at the interface is $\sim 3 \mathrm{~K}_{\mathrm{B}} \mathrm{T} / 4$ for an air interface. As such, wetting is an example of weak assembly, where the lowering in interaction energy for an oil molecule moving from the liquid-liquid interface to the liquid-solid interface is $\sim K_{B} T$, whereas the lowering in interaction energy for a water molecule moving to a hydrophilic surface is $>\mathrm{K}_{\mathrm{B}} \mathrm{T}$, due to both dispersion and polar interactions and is therefore an example of strong assembly. It is the difference in these energies that is used to effect oil/water separation using mesh membranes. The self-structuring of fluids at the interface can be devised to affect macroscopic separation of the fluids, provided the external forces are insufficient to overcome the capillary force on one phase, but not the other. For separating oilwater mixtures under gravity, a membrane that is superhydrophilic and superoleophobic (when submerged) will allow water to pass but provide a capillary barrier to the passage of oil, provided the hydrostatic head is insufficient to overcome the effect of the Laplace pressure that arises 
from the weak assembly of the oil molecules. A membrane of the other type (superhydrophobic and superoleophilic) will allow oil to pass and prevent the passage of water.

\subsection{Theory of superhydrophobicity and superhydrophilicity}

The wettability of a surface is usually quantified by the contact angle of a droplet of liquid on the surface. The droplet can either be in air or in another immiscible fluid. For smooth, homogenous, ideal surfaces the contact angle of a droplet on a surface immersed in another liquid is described by the balance of the interfacial energies in Young's equation;

$$
\gamma_{s, l}=\gamma_{s, d}+\gamma_{d, l} \operatorname{Cos} \theta_{\text {smooth }}
$$

where $s$, is the solid and is $d$ a liquid droplet immersed in a liquid $l$.

Surfaces are often described as hydrophilic or hydrophobic based on their contact angle with water in air on a smooth surface. There is no widely accepted definition, but a common one is that a hydropobic surface has a contact angle of $>90^{\circ}$ and consequently a hydrophilic surface has a contact angle $<90^{\circ}$. When the surfaces are rough more extreme wetting can be observed. Superhydrophilic surfaces arise when an innately hydrophilic surface is produced in a roughened form (such as from sintered particles) such that the contact angle is reduced to zero. The effect of roughness on the contact angle for an homogenous surface is described by the Wenzel equation, [12]

$$
r=\frac{\cos \theta_{\text {rough }}}{\cos \theta_{\text {smooth }}}
$$

where $r$, is the roughness parameter $(r>1)$, which is the ratio of the projected surface area of the rough surface to a perfectly flat surface. The contact angle of surfaces with oil is less often reported, as oils have low surface tension and therefore wet most flat surfaces. That is, surfaces 
are typically oleophilic. Therefore a superhydrophilic surface will typically also be a superoleophilic surface. However, when a superhydrophilic surface is first immersed in water it can be both superhydrophilic and (under water) superoleophobic as the water may fill the texture of the surface This is discussed further below.

Superhydrophobic surfaces can be produced from rough surfaces that are inherently hydrophobic. For a homogenous surface the Wenzel equation (equation 2) also applies, but as $\theta_{\text {smooth }}>90^{\circ}, \theta_{\text {rough }}>\theta_{\text {smooth. }}$ Additionally, gas can be trapped between the solid and the liquid phases forming a heterogenous composite interface, in which case the contact angle is given by a modified version of the Cassie equation, [13]

$$
\operatorname{Cos} \theta_{\text {Cassie }}=f_{1}\left[\operatorname{Cos} \theta_{\text {smooth }}+1\right]-1
$$

where $f_{l}$ is the fractional area of the surface (or three phase line) in contact with the liquid. For surfaces in which $\theta_{\text {smooth }}>90^{\circ}$, high effective contact angles result and as $f_{l}$ becomes smaller the contact angle approaches $180^{\circ}$. Superhydrophobic surfaces are usually also superoleophilic. Note that as oils do not give surfaces in which $\theta_{\text {smooth }}>90^{\circ}$ this approach will not work for producing superoleophobic surfaces. However, this can be achieved using surfaces engineered to have a reentrant structure $[14,15]$.

In membrane separation processes it is important to recognize that air is not present at the interface, that is, both of the fluid phases are liquids, and the desired properties are obtained when the wetting liquid penetrates the surface roughness, as air does in the Cassie state described above. This causes the non-wetting liquid to present with a very high (super) contact angle, as described by equation 4 where $f_{N W}$ is the fractional area of the surface (or three phase line) in 
contact with the non-wetting liquid and $\theta_{N W, s m o o t h}$ is the contact angle of the non-wetting fluid in the presence of the wetting fluid on a solid substrate.

$\operatorname{Cos} \theta_{N W, \text { Cassie }}=f_{N W}\left[\operatorname{Cos} \theta_{N W, \text { smooth }}+1\right]-1$

This heterogenous wetting state is favoured by very rough surfaces. The condition under which this occurs is given by[16];

$\operatorname{Cos} \theta_{W, \text { smooth }}>\frac{1-f_{W}}{r-f_{W}}$

where $f_{W}$ is the fractional area of the solid surface in contact with the wetting fluid. Thus a critical angle is defined such that when $\operatorname{Cos} \theta_{W, \text { smooth }}<\theta_{C}$, the wetting film will impregnate the textured or rough surface such that the non-wetting fluid has a very large contact angle. This is the preferred circumstance for mesh membranes, whether the wetting phase is the aqueous or oil phase as it results in a very high contact angle for the non-wetting phase. For an in depth discussion of wetting the interested reader is referred to the excellent monograph[16].

Membranes for oil/water separation are readily produced by treating a weave or a mesh to produce rough surfaces with the desired wetting properties. These mesh membranes can effectively separate immiscible oil/water mixtures and oil-in-water or water-in-oil emulsions, solely driven by gravity, with high permeation rates and separation efficiency. Most importantly, such mesh membranes exhibit good durability, stability and reusability. This, when combined with low cost and scalability, make them promising candidates for practical oil/water separations. In this manuscript, different mesh membranes with superhydrophobicity and superoleophilicity 
or superhydrophilicity and superoleophobicty induced by either surface modification or microand nanostructuring will be reviewed.

\subsection{Capillary basis for separation}

Mesh membranes use capillarity as the basis for selective permeability of oil and water. To achieve separation it is critical that one phase is allowed to pass readily through the membrane and the other phase is arrested. The wetting phase is readily able to traverse the membrane whereas, if the pores in a mesh membrane are sufficiently small compared to the droplet size, capillary pressure resists the passage of the non-wetting phase, see Fig 1. When the Laplace pressure of the non-wetting phase is sufficient to counteract external pressure (which is the hydrostatic pressure for gravity filtration) the non-wetting phase will not pass through the mesh. The Laplace pressure is larger when the curvature of the interface is greater. In an ideal twodimensional mesh made up of overlapping cylinders of radius $\mathrm{R}$ with an inter-cylinder separation space of 2D, this would be achieved when the applied pressure is less than the Laplace pressure of the non-wetting phase. As an approximation we assume that the minimal radius of curvature is where the pore is tightest for all contact angles. The pressure above which the non-wetting phase will pass through the membrane, called the breakthrough pressure, is then given by $\mathrm{P}_{\mathrm{B}}$,

$P_{B} \sim<\frac{-\gamma_{12} \operatorname{Cos} \theta_{N W, i}}{D}$ 
where $\gamma_{12}$ is the interfacial tension between the wetting phase and the non-wetting phase, $\theta_{N W, i}$ is the contact angle of the non-wetting phase on the solid surface when both are immersed in the wetting phase.

In some cases, such as electrospun materials deposited onto a mesh, meshes will consist of layers of fibres forming a three-dimensional structure. It is essential that the layers in a threedimensional mesh material for oil/water separation are spaced such that the re-entrant architecture of the mesh is maintained. This is an essential feature for any superoleophobic surface [17]. Even so the sagging of the interface can lead to breakthrough of the non-wetting phase. For a three dimensional mesh made up of overlapping cylinders of radius $\mathrm{R}$ with an intercylinder separation distance of 2D, the change in curvature of the non-wetting phase needs to be considered as it may cause the non-wetting phase to make contact with the next layer of the mesh, which will promote breakthrough. The breakthrough pressure that must not be exceeded is given below [18] (for a derivation see the appendix of Tuteja et al [19]).

$P_{B}<\frac{2 R \gamma_{12}}{D^{2}} \frac{1-\operatorname{Cos} \theta_{N W, i}}{1+2 R \operatorname{Sin} \theta_{N W, i} / D}$

This above expression provides clear criteria for the design of mesh membranes for oil-water separations. To increase the hydrostatic pressures required to cause breakthrough of the nonwetting phase, a large contact angle of the non-wetting phase and high interfacial tension between the liquid phases are required. In practice, the hydrostatic pressure does not increase the filtration rate as it is determined by the number of unblocked pores and the sedimentation rate of the wetting phase [18], so systems can be designed to produce low hydrostatic pressures without penalizing the flux. 
Cross flow filtration cells have been developed for microfiltration applications employing membranes, primarily as a means of limiting the development of thick filter cakes or blocking of pores that reduce the flux of filtrate through the membrane. Industrially, cross-flow filtration is employed as a continuous operation[20], which has the added advantage of limiting the increase in concentration of the rejected phase in the solution being filtered. Cross flow cells are also ideally suited to mesh membrane applications but for different reasons. In the case of mesh membranes for the separation of two fluids, the development of a filter cake is generally not an important consideration. However cross flow filtration cells mounted horizontally meet both the need to limit the hydrostatic head to prevent breakthrough and minimise the concentration of the non-wetting phase in the solution being filtered, in order to maximize the filtration rate of mesh membranes[21]. Pore blocking is a consideration for oil-water separation using mesh membranes but this is primarily a function of the concentration of droplets of the non-wetting phase as the extreme wettability of the membranes inhibits conventional fouling when only liquids are present. Singh et al have discussed a range of models for describing the flux through a membrane used to separate oily emulsions using cross-flow[22]. Important operational parameters are the cross flow rate, the trans-membrane pressure and the Reynolds number[21]. For mesh membrane applications the combined trans-membrane pressure and the hydrostatic pressure have to be less than the breakthrough pressure.

Provided the necessary extreme and stable wetting properties can be engineered on a membrane of appropriate geometry, the material used for the membrane is not important for the separation process. Rather, other considerations such as cost, anti-fouling properties, chemical stability, ease of fabrication, temperature stability, pore size and the ability to prepare the membrane on a 
large scale will govern the choice of membrane material. When considering the method of fabrication it should be recognized that for a given architecture of fibres of radius R, and fibre separation distance of 2D, in any practical application a single layer mesh (two-dimensional architecture) such as a stainless steel mesh should outperform a fibre mat (three dimensional architecture) as sagging of the interface can lead to breakthrough in the latter case.

\section{Materials for coating mesh membranes for oil-water separation}

\subsection{Polymers}

The wettability of solid surfaces is dominated by the morphology and chemical composition of the surfaces [23]. Currently, the most effective way to create a surface with extreme wettability is by coating hierarchical structures with micro and nano roughness with chemicals that lead to a low surface-energy [24]. Fluorinated and silanated polymers are thus attractive for fabricating separation membranes. To date, a series of polymer based mesh membranes with extreme wettability have been produced using, PTFE [25], polyacrylamide (PAM) [26], poly(sodium methacrylate) (PNaMA) [27], polydimethylsiloxane (PDMS) [28], poly(sulfobetaine methacrylate) (PSBMA)/polydopamine (PDA) [29] and fluorodecyl polyhedral oligometric silsesquioxiane $+x$-poly(ethylene glycol) diacrylate (POSS $+x$-PEGDA) [18]. Fluorodecyl POSS $+x$-PEGDA coated mesh membranes fabricated by dip coating and drying under nitrogen showed stable superhydrophilicity and superoleophobicity in air and water [18]. As illustrated in Fig. 2, after coating with a layer of fluorodecyl POSS the x-PEGDA surface is completely covered with crystalline domains of fluorodecyl POSS, indicating substantial surface segregation of the fluorodecyl POSS molecules, which are expected to migrate over the surface 
upon exposure to a wetting phase and thereby produce an extremely low energ surface [17]. Because of the extreme surface wettability, this membrane can separate a range of different oil and water mixtures in a single-unit operation with high separation efficiency, over $99.9 \%$, by using the difference in capillary forces acting on the two phases [18].

PAM hydrogel based mesh membranes prepared via a light-initiated polymerization process exhibited stable superhydrophilicity and underwater superoleophobicity with a contact angle (CA) of $153 \pm 1.8^{\circ}$ and low oil adhesion of less than $5 \mu \mathrm{N}$ [26]. Here, apart from the superoleophobicity in air, the underwater superoleophobicity is achieved by imibition of the water phase into the micro or nano hierarchical structure of the polymer membrane coating. When the oil droplets make contact with the polymer based coatings, water remains trapped in the rough micro and nanostructures, forming an oil/water/solid composite interface that greatly decreases the contact area between the oil droplet and the solid surface $[30,31]$. Thus the oiladhesion of the surface is extremely low, mimicking the approach that the pitcher plant uses to trap insects that has been adopted for producing antifouling surfaces [32]. This membrane shows a separation efficiency over $99 \%$ and good resistance to oil fouling [26].

\subsection{Boron nitride nanotubes}

Boron nitride nanotubes (BNNTs) possess remarkable mechanical properties, high thermal conductivity, outstanding electrical insulation, excellent chemical stability, as well as superb resistance to oxidation [33]. Additionally, BNNT films are poorly wet by water due to adsorbed hydrocarbon contamination and nanometer scale roughness [34, 35]. This water repellency can be further enhanced by partial perpendicular alignment of the BNNTs to the substrate, resulting in superhydrophobicity with a water contact angle (CA) over $150^{\circ}$ and reduced $\mathrm{CA}$ hysteresis 
[36]. These properties make BNNTs attractive candidates for use as superhydrophobic surfaces in harsh chemical and thermal conditions. As shown in Fig. 3, Chen's group fabricated a BNNT coated membrane using a vapor-liquid-solid growth followed by high temperature annealing process [37]. This simple, scalable and inexpensive process produced highly dense and pure BNNTs with diameters in the range of 100 to $400 \mathrm{~nm}$ that were partially aligned perpendicularly to the stainless steel mesh substrate, producing a superhydrophobic $\left(\mathrm{CA}>150^{\circ}\right)$ and superoleophilic $\left(\mathrm{CA}<1{ }^{\circ}\right)$ surface, almost without hysteresis, as shown in Fig. 4 [37]. Due to this special surface wettability, BNNT based mesh membranes can be practically used as a highly efficient filtration membrane for the separation of oil and water. Importantly, the BNNTs are strongly adhered to the stainless steel mesh substrates[35], offering the necessary stability and durability for practical applications.

\subsection{Metal oxides and hydroxides}

Metal oxides and hydroxides are normally hydrophilic, but if the surface is rough or textured surfaces and coated with a low energy material it becomes superhydrophobic [9, 38-45]. Recently, a series of metal oxide and hydroxide materials such as $\mathrm{Cu}_{2} \mathrm{O}$ [40], $\mathrm{TiO}_{2}[39,45], \mathrm{ZnO}$ $[38,44], \mathrm{NiOOH}[41]$ and $\mathrm{Cu}(\mathrm{OH})_{2}[9,42,43]$, have been successfully fabricated onto mesh membranes. An example is the superhydrophilic and underwater superoleophobic $\mathrm{Cu}(\mathrm{OH})_{2}$ coated mesh membrane prepared by Jin's group via surface oxidation of a copper mesh in alkaline aqueous solution [9]. After 30 minutes of oxidation, $\mathrm{Cu}(\mathrm{OH})_{2}$ nanowires with lengths in the range of $10-15 \mu \mathrm{m}$ and diameters in the range of $200-500 \mathrm{~nm}$ were formed, which grow perpendicular to the mesh walls and intertwine with each other, as shown in Fig. 5 [9]. These surfaces exhibit an underwater superoleophobicity for oils $\left(\mathrm{CA}>150^{\circ}\right)$ and an ultralow adhesion 
force (below $1 \mu \mathrm{N}$ ) [9]. This surface wettability and ultralow oil adhesion can be attributed to the micro/nanohierarchical rough structure arising from the long and densely arrayed $\mathrm{Cu}(\mathrm{OH})_{2}$ nanowires on the copper mesh and the hydrophilic nature of $\mathrm{Cu}(\mathrm{OH})_{2}$, such that when immersed in water, water is trapped in the hierarchical structure to form an oil/water/solid interface in the presence of oil [9]. This membrane can effectively separate immiscible oil/water mixtures even some oil-in-water emulsions with extremely high separation efficiency (residual oil in the filtrate after only one pass was lower than $30 \mathrm{ppm}$ ) and flux over $1 \times 10^{5} \mathrm{Lm}^{-2} \mathrm{~h}^{-1}$. Most importantly, the membrane exhibited excellent anti-oil fouling properties and superior stability, even in strong alkaline conditions $(\mathrm{pH} \geq 14)$ under long term use [9].

Lin's group developed a novel double layer $\mathrm{TiO}_{2}$-based mesh membrane and realized the separation of oil from water and the degradation of soluble pollutants in one device [39]. Both layers of $\mathrm{TiO}_{2}$ coated meshes were prepared by a simple hydrothermal method. The upper layer consisted of only $\mathrm{TiO}_{2}$ coated mesh, but the lower layer of $\mathrm{TiO}_{2}$ was modified with octadecylphosphonic acid to impart it with superhydrophobicity and superoleophilicity [39]. The membrane exhibited not only good selectivity but also excellent photocatalytic abilities. Such a membrane can be successfully used for the separation of insoluble oil from water due to its extreme wettability, as well as the degradation of soluble pollutants in water under UV light because of the photocatalytic abilities of $\mathrm{TiO}_{2}[39]$.

\subsection{Graphene oxide}

The inherent hydrophilic nature of oxygen-containing functional groups, such as carboxyl and hydroxyl groups, on the basal plane and at the edges of graphene oxide (GO) makes it an 
ideal candidate for the fabrication of various underwater superoleophobic mesh membranes that can be used for oil and water separation [46, 47]. Hydrophilic GO can be readily coated on a stainless steel mesh through a simple dip-coating process or a drop-coating followed by $\mathrm{O}_{2}$ plasma treatment (as shown in Fig. 6) [46]. The mesh membranes coated with GO effectively trap water and exhibit underwater superoleophobicity. Due to this extreme wettability, various oils can be simply and efficiently separated from water under gravity with a high efficiency and flux [46]. Importantly, the water trapped in the rough nanostructures provides a strong repulsive force for oils, not only resulting in greater oil contact angles, but also lowering the oil-adhesion force [46]. Therefore, such low adhesion can effectively prevent GO coated meshes from fouling during the oil-water separation processes.

\subsection{Diamond-like carbon}

Due to the excellent physical and chemical properties, such as good mechanical stability, extreme hardness, high corrosion resistance, and biocompatibility, as well as the tunability of the surface wettability and adhesion, diamond-like carbon (DLC) can also be used as a superhydrophobic coating on a mesh membrane surface for oil and water separation [48, 49]. Cortese's group developed a novel superhydrophobic and superoleophilic DLC coated cotton textile by one-step growth of a DLC film using plasma-enhanced chemical vapor deposition (Fig. 7) [49]. The morphology and wettability of the DLC coating can be adjusted by varying the plasma pre-treatment time of the cotton. The highest water $\mathrm{CA}$ of about $170^{\circ}$ was obtained after 60 min pre-treatment, as shown in Fig. 7 [49]. The reusable DLC coated cotton textiles were

effective at separating oil and water mixtures under gravity with high separation efficiency and demonstrated selective oil absorption capacity [49]. 


\subsection{Metals}

The surface wettability of pure metals can be altered by surface modification and increasing the roughness [50]. Pure Ag and $\mathrm{Cu}$ nanoparticle based mesh membranes have been fabricated for oil and water separation [50-52]. Ag coated meshes were prepared by a simple immersion of copper meshes into an aqueous solution of $\mathrm{AgNO}_{3}$ at room temperature (Fig. 8) [50]. The obtained Ag-coated mesh surface exhibited extreme wetting with a contact angle of $164 \pm 1.4^{\circ}$ and a sliding angle of $4 \pm 0.8^{\circ}$ for water, and a contact angle of $0^{\circ}$ for kerosene [50]. Here, carbon contaminants from ambient air are spontaneously adsorbed onto the silver surface resulting in superhydrophobicity and superoleophilicity [50]. This Ag nanoparticle based mesh membrane separated oil from water with efficiencies in excess of $92 \%$ in repeated cycles [50]. Furthermore, the membrane possessed excellent resistance to corrosion and was stable at temperatures ranging from -20 to $250{ }^{\circ} \mathrm{C}$. Superhydrophobic and superoleophilic $\mathrm{Cu}$ nanoparticle based mesh membranes can be prepared by electrodeposition with a subsequent modification process $[51,52]$. These $\mathrm{Cu}$ nanoparticle based membranes effectively and rapidly separate oil and water. Moreover, these mesh membranes exhibit strong stability as the $\mathrm{Cu}$ nanoparticles can bear external pressure, high temperature, and long-term immersion in nonpolar solvent [51].

\subsection{Zeolites}

Zeolite films have uniform pore size, good thermal and mechanical stability and excellent chemical resistance to acids and salt solutions [53]. Recently, zeolite coated meshes with underwater superoleophobicity have been successfully fabricated by hydrothermal synthesis, as shown in Fig. 9 [53, 54]. Various oils can be separated from water with these zeolite based mesh membranes under gravity with a high flux of $9 \times 10^{4} \mathrm{Lm}^{-2} \mathrm{~h}^{-1}$ and high efficiency (less than 6 
ppm oil) [53]. In addition, the separation flux is tunable by changing the pore size which depends on the crystallization time of the zeolite crystals [53]. More importantly, zeolite based mesh membranes are recyclable by calcination owing to the high thermal stability of zeolite crystals. Further, they are chemically stable in different corrosive conditions [53]. These properties make zeolite based mesh membranes very attractive in harsh environments.

\section{Choice of membrane material}

The excellent separation performance of mesh membranes achieved in the laboratory is now being applied to a large scale in practical applications. An example is Ferrocep ${ }^{\circledR}$ membranes that utilize a stainless steel mesh coated with titania particles which are used for the removal of oil, grease and fat in a range of applications. We expect that mesh membranes will be applied to many other applications provided membrane production can be scaled up at a suitable cost and that membrane dimensions are reduced to handle the emulsions with small droplet sizes that are more difficult to separate[20]. Additionally, consideration must be given to the effectiveness of mesh membranes in the presence of contaminants and foulants, such as asphaltenes and waxes that are present in crude oil, in many applications. Insufficient laboratory studies focusing on testing and producing mesh membranes resistant to contaminants have been performed to determine the significance of this challenge, though work has shown that polymer coated, metal oxide and graphene oxide mesh membranes exhibit very low adhesion for oil droplets, suggesting this may not be a significant obstacle to practical application. Mesh membranes are unable to reject droplets that are smaller than the pore diameter of the coated meshed. In general mesh membranes employed in laboratory studies have large pore diameters of 100 microns or more. In practical applications droplets of 50 microns or greater are generally very unstable and 
coalesce, whereas droplets smaller than 10 microns are highly stable and therefore pose a greater separation challenge[20, 22]. Thus many practical applications will require mesh membranes with smaller pore diameters in order to effectively separate small droplets. Laboratory studies have paid little attention to the challenge of separating small droplets.

The choice of material for any particular separation process will be governed by a large number of factors including chemical stability, temperature requirements and mechanical strength. We expect that graphene Oxide, BNNT and Diamond like Carbon coated membranes will be difficult to produce on a large scale. Polymer coated membranes generally meet most requirements but are not suitable for high temperature environments. Whilst metals and metal oxides can withstand high temperatures they are not suited to some chemical environments. Zeolites are robust and can withstand high temperatures and harsh conditions but the process of coating meshes with zeolites may prove to be challenging on an industrial scale.

\section{Conclusions}

A wide-range of materials have been employed for the production of mesh membranes for the separation of oil and water. The basic requirement is that the system must exhibit extreme wettability, whereby a membrane with superhydrophobicity and superoleophilicity or underwater superhydrophilicity and superoleophobicty is fabricated by creating a layer of hierarchical structures on the nano and micro scale, on mesh materials. This is followed by modification with low surface-energy chemicals to produce a membrane with superhydrophobicity and superoleophilicity or the introduction of water into the hydrophilic hierarchical structure to produce a membrane that exhibits underwater superhydrophilicity and superoleophobicty. These membranes can effectively separate immiscible oil/water mixtures solely driven by gravity, with 
high separation speed and efficiency. More importantly, such mesh membranes exhibit good durability, stability and reusability. Different membrane materials offer a choice of secondary properties such as mechanical strength, heat resistance, chemical stability and electrical resistance. The choice of membrane material will ultimately be determined by considerations of the chemical environment in which the membrane will operate, the cost of production, the scalability of the preparation process and particular specialized requirements.

\section{Acknowledgements}

YL and ZL acknowledge KAUST Center competitive grant FCC/1/1972-16-01. HC and VC acknowledge the Australian Research Council (ARC)'s support in the forms of Discovery Projects DP1095998, DP140102371 and Postdoctoral Research Fellowship DP1095998 and Future Fellowship programs FT 100100449.

\section{References}

[1] Asatekin A, Mayes AM. Oil industry wastewater treatment with fouling resistant membranes containing amphiphilic comb copolymers. Environmental science \& technology. 2009;43:4487-92.

[2] Gossen L, Velichkina L. Environmental problems of the oil-and-gas industry (Review). Petroleum Chemistry. 2006;46:67-72.

[3] Lahann J. Environmental nanotechnology: Nanomaterials clean up. Nature nanotechnology. 2008;3:320-1.

[4] Ruan C, Ai K, Li X, Lu L. A superhydrophobic sponge with excellent absorbency and flame retardancy. Angewandte Chemie International Edition. 2014;53:5556-60.

[5] Cheryan M, Rajagopalan N. Membrane processing of oily streams. Wastewater treatment and waste reduction. Journal of Membrane Science. 1998;151:13-28.

[6] Elmaleh S, Ghaffor N. Cross-flow ultrafiltration of hydrocarbon and biological solid mixed suspensions. Journal of Membrane Science. 1996;118:111-20.

[7] Shannon MA, Bohn PW, Elimelech M, Georgiadis JG, Marinas BJ, Mayes AM. Science and technology for water purification in the coming decades. Nature. 2008;452:301-10.

[8] Kerr RA. An audacious decision in crisis gets cautious praise. Science. 2010;329:735-6. 
[9] Zhang F, Zhang WB, Shi Z, Wang D, Jin J, Jiang L. Nanowire - Haired Inorganic Membranes with Superhydrophilicity and Underwater Ultralow Adhesive Superoleophobicity for High - Efficiency Oil/Water Separation. Advanced Materials. 2013;25:4192-8.

[10] Tirmizi NP, Raghuraman B, Wiencek J. Demulsification of water/oil/solid emulsions by hollowfiber membranes. Aiche J. 1996;42:1263-76.

[11] Angelescu DE, Mercier B, Siess D, Schroeder R. Microfluidic Capillary Separation and Real-Time Spectroscopic Analysis of Specific Components from Multiphase Mixtures. Analytical Chemistry. 2010;82:2412-20.

[12] Wenzel RN. Resistance of solid surfaces to wetting by water. Ind Eng Chem. 1936;28:988-94.

[13] Cassie ABD, Baxter S. Wettability of porous surfaces. Trans Fraday Soc. 1944;40:546-51.

[14] Tuteja A, Choi W, Ma ML, Mabry JM, Mazzella SA, Rutledge GC, et al. Designing superoleophobic surfaces. Science. 2007;318:1618-22.

[15] Marmur A. From hygrophilic to superhygrophobic: Theoretical conditions for making highcontact-angle surfaces from low-contact-angle materials. Langmuir. 2008;24:7573-9.

[16] Pierre-Gilles dG, Brichard-Wyart F, Quere D. Capillarity and Wetting Phenomena. New York: Springer; 2002.

[17] Tuteja A, Choi W, Ma M, Mabry JM, Mazzella SA, Rutledge GC, et al. Designing superoleophobic surfaces. Science. 2007;318:1618-22.

[18] Kota AK, Kwon G, Choi W, Mabry JM, Tuteja A. Hygro-responsive membranes for effective oilwater separation. Nature communications. 2012;3:1025.

[19] Tuteja A, Choi W, Mabry JM, McKinley GH, Cohen RE. Robust omniphobic surfaces. Proceedings of the National Academy of Sciences of the United States of America. 2008;105:18200-5.

[20] Chakrabarty B, Ghoshal AK, Purkait MK. Cross-flow ultrafiltration of stable oil-in-water emulsion using polysulfone membranes. Chemical Engineering Journal. 2010;165:447-56.

[21] Koltuniewicz AB, Field RW, Arnot TC. Cross-flow and dead-end microfiltration of oily-water emulsion .1. Experimental-study and analysis of flux decline. Journal of Membrane Science. 1995;102:193-207.

[22] Singh V, Purkait MK, Das C. Cross-Flow Microfiltration of Industrial Oily Wastewater: Experimental and Theoretical Consideration. Separation Science and Technology. 2011;46:1213-23. [23] Feng L, Li S, Li Y, Li H, Zhang L, Zhai J, et al. Super - hydrophobic surfaces: from natural to artificial. Advanced materials. 2002;14:1857-60.

[24] Howarter JA, Youngblood JP. Amphiphile grafted membranes for the separation of oil-in-water dispersions. J Colloid Interface Sci. 2009;329:127-32.

[25] Feng L, Zhang Z, Mai Z, Ma Y, Liu B, Jiang L, et al. A super - hydrophobic and super - oleophilic coating mesh film for the separation of oil and water. Angewandte Chemie International Edition. 2004;43:2012-4.

[26] Xue Z, Wang S, Lin L, Chen L, Liu M, Feng L, et al. A novel superhydrophilic and underwater superoleophobic hydrogel - coated mesh for oil/water separation. Advanced Materials.

2011;23:4270-3.

[27] Dunderdale GJ, Urata C, Sato T, England MW, Hozumi A. Continuous, High-speed and Efficient Oil/Water Separation using Meshes with Antagonistic Wetting Properties. ACS Appl Mater Interfaces. 2015;7:18915-9.

[28] Crick CR, Gibbins JA, Parkin IP. Superhydrophobic polymer-coated copper-mesh; membranes for highly efficient oil-water separation. Journal of Materials Chemistry A. 2013;1:5943-8.

[29] Ren P-F, Yang H-C, Jin Y-N, Liang H-Q, Wan L-S, Xu Z-K. Underwater superoleophobic meshes fabricated by poly (sulfobetaine)/polydopamine co-deposition. RSC Advances. 2015;5:47592-8.

[30] Lin L, Liu M, Chen L, Chen P, Ma J, Han D, et al. Bio - Inspired Hierarchical MacromoleculeNanoclay Hydrogels for Robust Underwater Superoleophobicity. Advanced Materials. 2010;22:4826-30. 
[31] Liu M, Wang S, Wei Z, Song Y, Jiang L. Bioinspired design of a superoleophobic and low adhesive water/solid interface. Advanced Materials. 2009;21:665-9.

[32] Wong TS, Kang SH, Tang SKY, Smythe EJ, Hatton BD, Grinthal A, et al. Bioinspired self-repairing slippery surfaces with pressure-stable omniphobicity. Nature. 2011;477:443-7.

[33] Golberg D, Bando Y, Tang C, Zhi C. Boron nitride nanotubes. Advanced Materials. 2007;19:2413-32.

[34] Li L, Li LH, Ramakrishnan S, Dai XJ, Nicholas K, Chen Y, et al. Controlling wettability of boron nitride nanotube films and improved cell proliferation. The Journal of Physical Chemistry C.

2012;116:18334-9.

[35] Li LH, Chen Y, Glushenkov AM. Synthesis of boron nitride nanotubes by boron ink annealing. Nanotechnology. 2010;21:105601.

[36] Lee CH, Drelich J, Yap YK. Superhydrophobicity of boron nitride nanotubes grown on silicon substrates. Langmuir. 2009;25:4853-60.

[37] Yu Y, Chen H, Liu Y, Craig V, Li LH, Chen Y. Superhydrophobic and Superoleophilic Boron Nitride Nanotube - Coated Stainless Steel Meshes for Oil and Water Separation. Advanced Materials Interfaces. 2014;1:1300002.

[38] Du X, Huang X, Li X, Meng X, Yao L, He J, et al. Wettability behavior of special microscale ZnO nail-coated mesh films for oil-water separation. J Colloid Interface Sci. 2015;458:79-86.

[39] Gao C, Sun Z, Li K, Chen Y, Cao Y, Zhang S, et al. Integrated oil separation and water purification by a double-layer TiO 2-based mesh. Energy \& Environmental Science. 2013;6:1147-51.

[40] Kong L-H, Chen X-H, Yu L-G, Wu Z-S, Zhang P-Y. Superhydrophobic Cuprous Oxide

Nanostructures on Phosphor-Copper Meshes and Their Oil-Water Separation and Oil Spill Cleanup. ACS Appl Mater Interfaces. 2015;7:2616-25.

[41] Li J, Cheng HM, Chan CY, Ng PF, Chen L, Fei B, et al. Superhydrophilic and underwater superoleophobic mesh coating for efficient oil-water separation. RSC Advances. 2015;5:51537-41. [42] Liu N, Cao Y, Lin X, Chen Y, Feng L, Wei Y. A facile solvent-manipulated mesh for reversible oil/water separation. ACS Appl Mater Interfaces. 2014;6:12821-6.

[43] Pan Q, Wang M, Wang H. Separating small amount of water and hydrophobic solvents by novel superhydrophobic copper meshes. Applied Surface Science. 2008;254:6002-6.

[44] Wang C-F, Tzeng F-S, Chen H-G, Chang C-J. Ultraviolet-durable superhydrophobic zinc oxidecoated mesh films for surface and underwater-oil capture and transportation. Langmuir.

2012;28:10015-9.

[45] Zhang L, Zhong Y, Cha D, Wang P. A self-cleaning underwater superoleophobic mesh for oilwater separation. Scientific reports. 2013;3:2326.

[46] Dong Y, Li J, Shi L, Wang X, Guo Z, Liu W. Underwater superoleophobic graphene oxide coated meshes for the separation of oil and water. Chemical Communications. 2014;50:5586-9.

[47] Liu Y-Q, Zhang Y-L, Fu X-Y, Sun H-B. Bioinspired underwater superoleophobic membrane based on graphene oxide coated wire mesh for efficient oil/water separation. ACS Appl Mater Interfaces. 2015;7:20930-6.

[48] Caschera D, Cortese B, Mezzi A, Brucale M, Ingo GM, Gigli G, et al. Ultra

hydrophobic/superhydrophilic modified cotton textiles through functionalized diamond-like carbon coatings for self-cleaning applications. Langmuir. 2013;29:2775-83.

[49] Cortese B, Caschera D, Federici F, Ingo GM, Gigli G. Superhydrophobic fabrics for oil-water separation through a diamond like carbon (DLC) coating. Journal of Materials Chemistry A.

2014;2:6781-9.

[50] Wang F, Lei S, Xu Y, Ou J. Green Approach to the Fabrication of Superhydrophobic Mesh Surface for Oil/Water Separation. ChemPhysChem. 2015;16:2237-43.

[51] Wang B, Guo Z. Superhydrophobic copper mesh films with rapid oil/water separation properties by electrochemical deposition inspired from butterfly wing. Applied Physics Letters. 2013;103:063704. 
[52] Wang S, Song Y, Jiang L. Microscale and nanoscale hierarchical structured mesh films with superhydrophobic and superoleophilic properties induced by long-chain fatty acids.

Nanotechnology. 2007;18:015103.

[53] Zeng J, Guo Z. Superhydrophilic and underwater superoleophobic MFI zeolite-coated film for oil/water separation. Colloids and Surfaces A: Physicochemical and Engineering Aspects.

2014;444:283-8.

[54] Wen Q, Di J, Jiang L, Yu J, Xu R. Zeolite-coated mesh film for efficient oil-water separation. Chemical Science. 2013;4:591-5. 
Figures
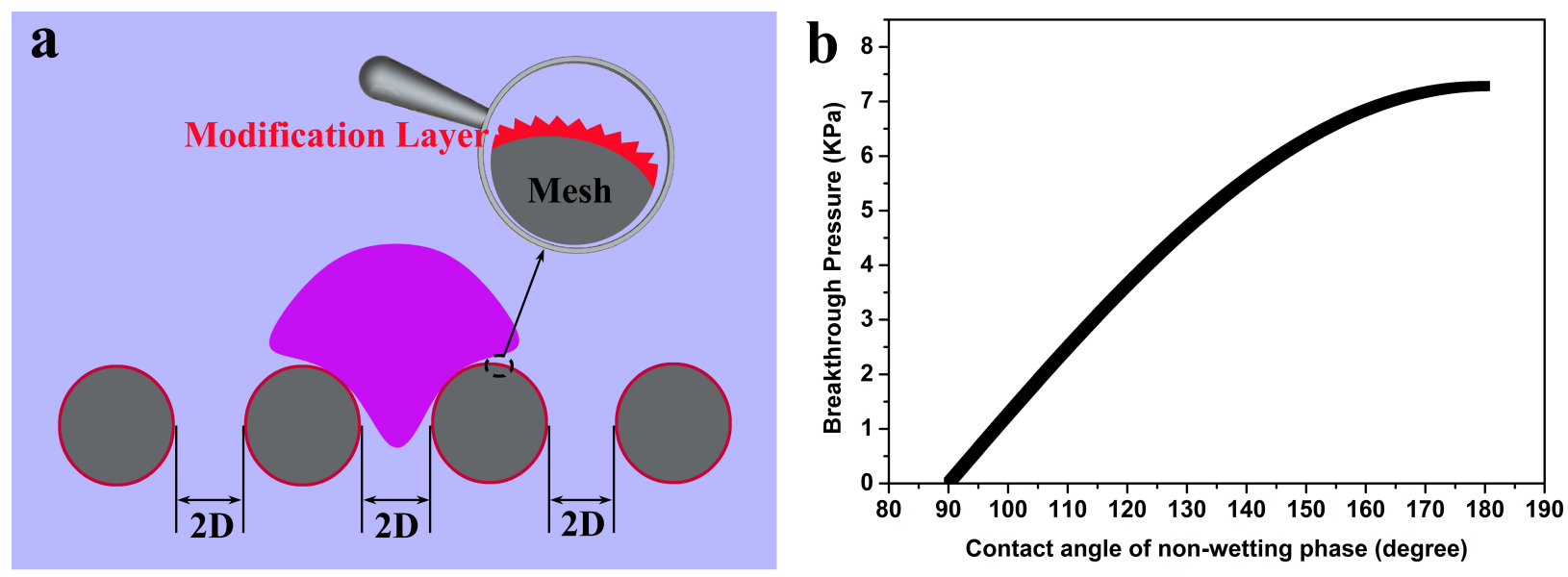

Fig. 1. (a) Schematic of a 2D superhydrophilic and superoleophobic mesh (grey) showing the rejection of an oil droplet (orange) due to capillary forces through the pores of radius D. (b) Breakthrough pressure for a 2D mesh with pore radius of 5 microns as a function of the contact angle of the non-wetting oil on the mesh immersed in water calculated using equation 6. 

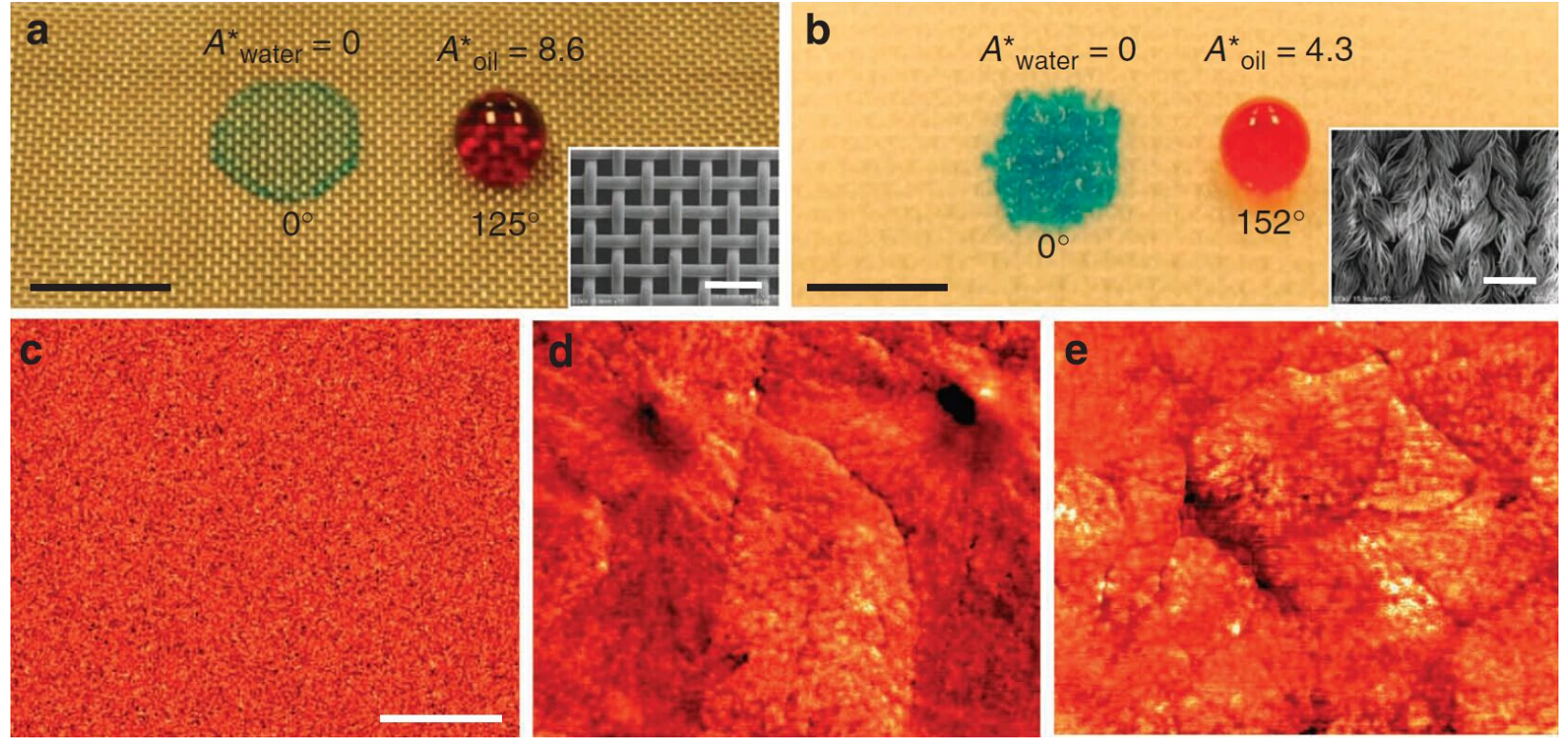

Fig. 2. (a, b) Droplets of water (dyed blue) and rapeseed oil (dyed red) on coated stainless steel mesh 100 (a) and coated polyester fabric (b). Both surfaces have been dip-coated with a $20 \mathrm{wt} \%$ fluorodecyl POSS + $\mathrm{x}$-PEGDA blend. Scale bars: $5 \mathrm{~mm}$. Insets, morphologies of the respective dip-coated mesh and fabric surfaces. Scale bars: $500 \mu \mathrm{m}$. (c-e) AFM phase images of surfaces coated with x-PEGDA (c), a $10 \mathrm{wt} \%$ fluorodecyl POSS $+\mathrm{x}$-PEGDA blend (d) and a $20 \mathrm{wt} \%$ fluorodecyl POSS $+\mathrm{x}-\mathrm{PEGDA}$ blend (e). The phase angle ranges are $0^{\circ}-115^{\circ}(\mathrm{c}), 0^{\circ}-25^{\circ}(\mathrm{d})$ and $0^{\circ}-$ $21^{\circ}$ (e). Scale bar: $2 \mu \mathrm{m}$. 


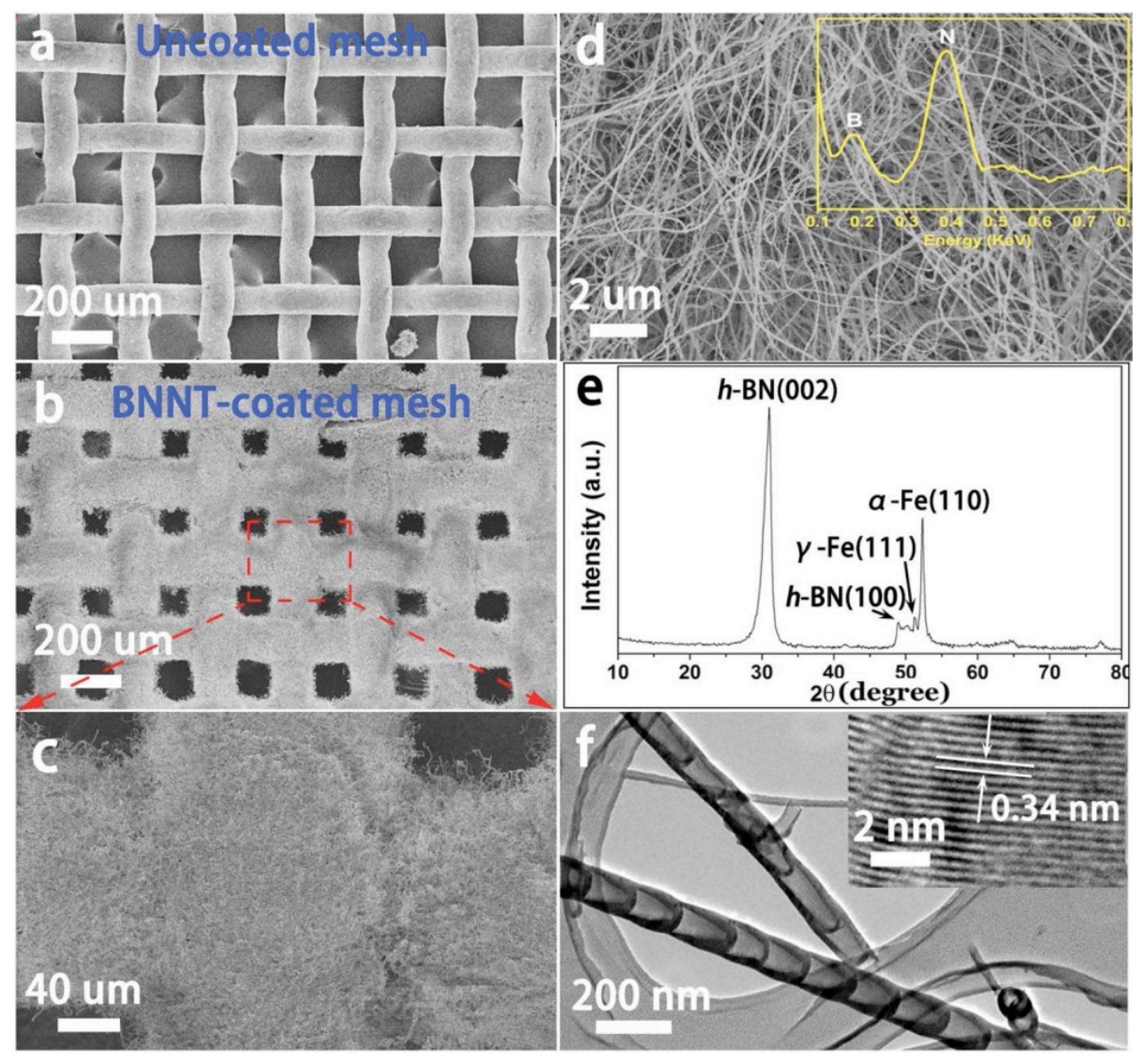

Fig. 3. FESEM images of the (a) uncoated and (b) boron nitride nanotube (BNNT) coated stainless steel meshes, (c) the BNNTs on the mesh surface, (d) the corresponding BNNTs removed from the surface. (e) XRD pattern and (f) TEM image of the BNNTs. The insets in (d) and (f) are the EDS spectrum and high-resolution TEM image of the BNNTs, respectively. 


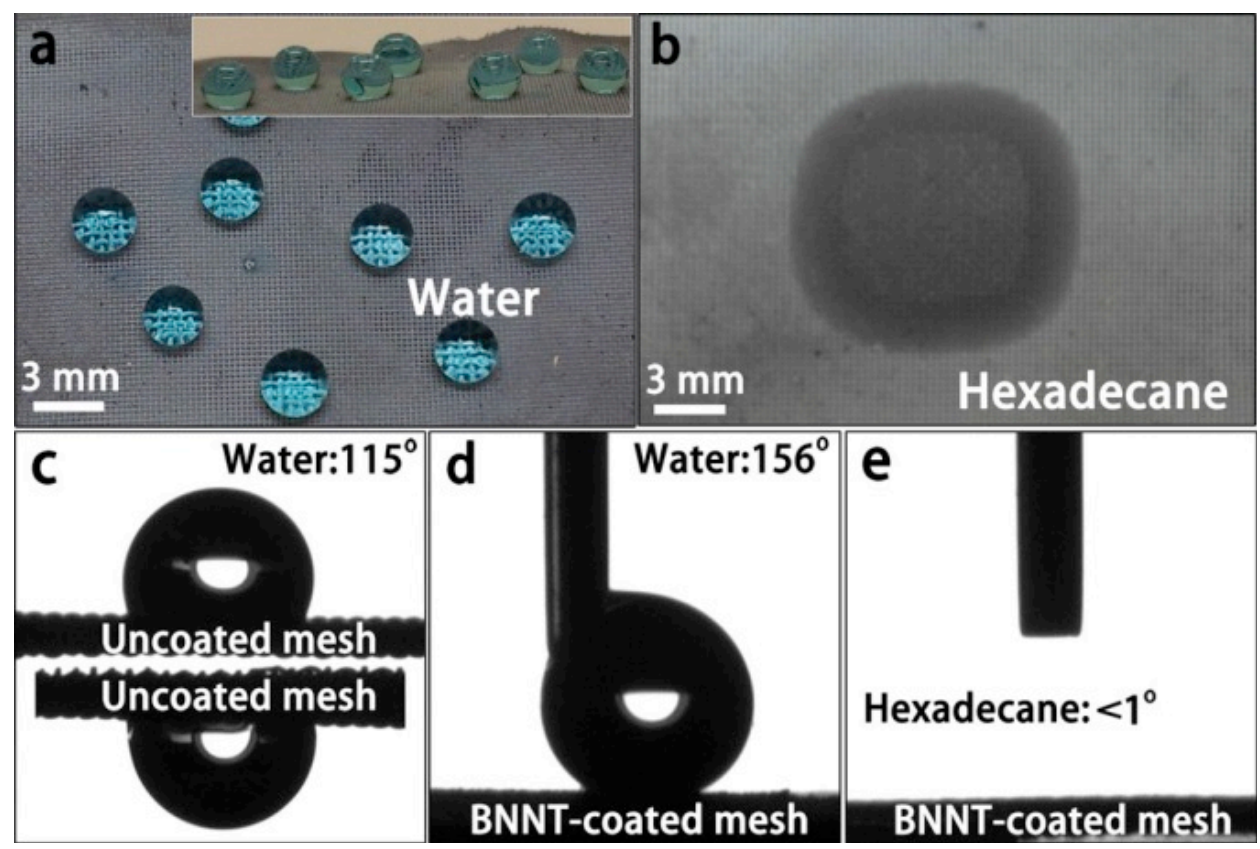

Fig. 4. (a) Photograph of water droplets (dyed with methylene blue) on the surface of a Boron Nitride Nanotube coated mesh (inset: a side view of a water droplet); (b) photograph of an oil droplet completely spread on the coated mesh surface; (c) shapes of water droplets $(6 \mu \mathrm{L})$ on and adhering to an uncoated mesh (CA of $\left.115^{\circ}\right)$; (d) shapes of water droplets $(6 \mu \mathrm{L})$ on a coated mesh $\left(\mathrm{CA}\right.$ of $\left.156^{\circ}\right)$; (e) photograph of the hexadecane droplet on the coated mesh surface with a CA of nearly zero. 

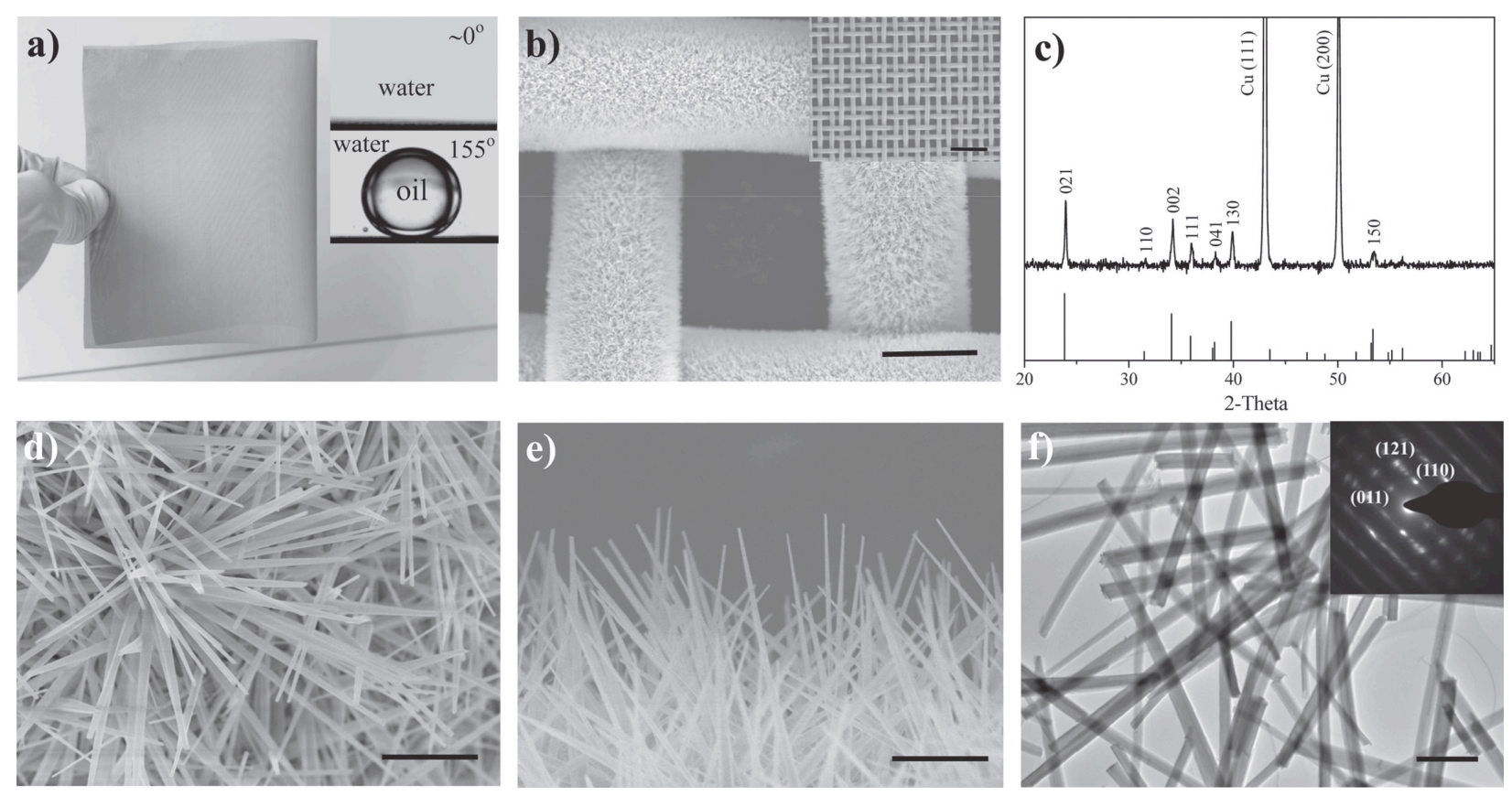

Fig. 5. Characterisation of nanowire-haired membrane on a copper mesh after a reaction time of $30 \mathrm{~min}$. (a)

Photograph of a large-scale nanowire haired membrane. The insets are photographs of a water droplet on the membrane showing nearly zero contact angle (upper) and an underwater oil droplet $(3 \mu \mathrm{L}, 1,2$-dichloroethane) on the membrane showing contact angle of $155^{\circ}$ (lower). (b) SEM image of nanowire-haired membrane (scale bar: 50 $\mu \mathrm{m})$. The inset is a low-magnification SEM image with scale bar of $200 \mu \mathrm{m}$ ). (c) XRD spectrum of the nanowire haired membrane. Below is the corresponding diffraction peaks of the $\mathrm{Cu}(\mathrm{OH})_{2}$ standard card. (d) and (e) Top-view and cross-section SEM images of the nanowire-haired membrane showing the nanowires growing out from the membrane (scale bar: $5 \mu \mathrm{m}$ ). (f) TEM image of nanowires. For TEM characterization, a nanowire haired membrane was treated by ultrasonification to peel off nanowires from the membrane (scale bar: $1 \mu \mathrm{m})$. 


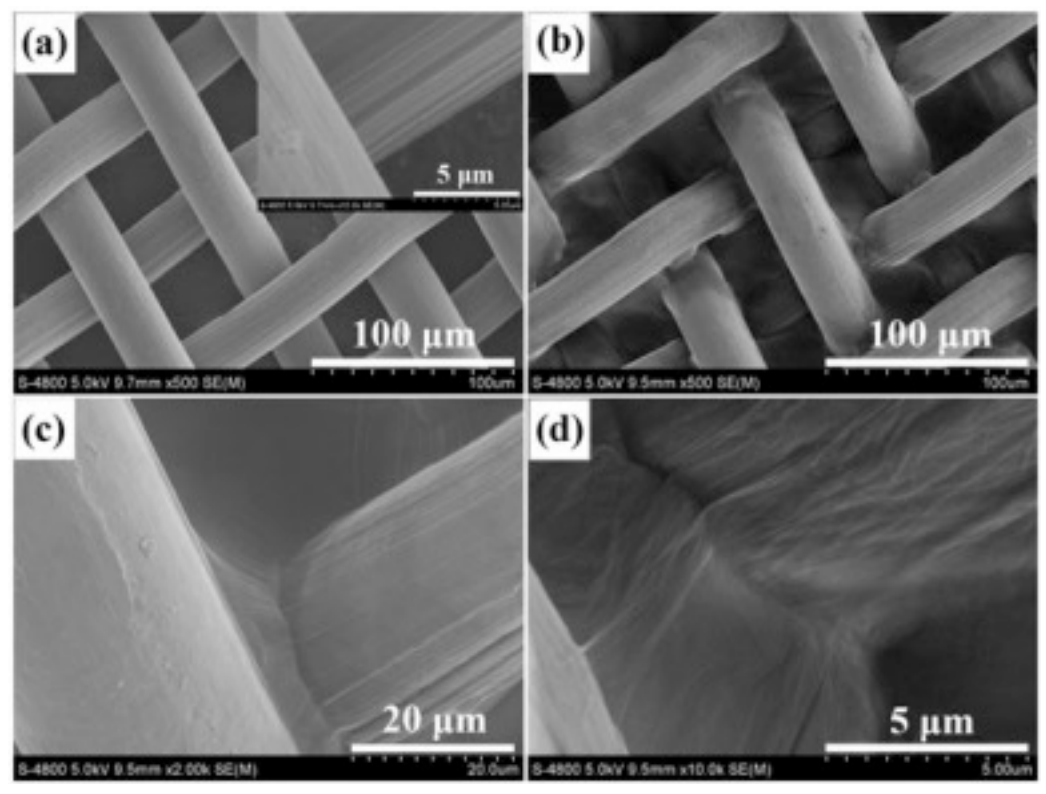

Fig. 6. SEM images of an uncoated stainless steel mesh (a) and graphene oxide coated meshes (b-d) with an average pore diameter of about $38 \mathrm{~mm}$. The inset in (a) shows the magnified view of the smooth surface of an uncoated mesh. 
a)

b)
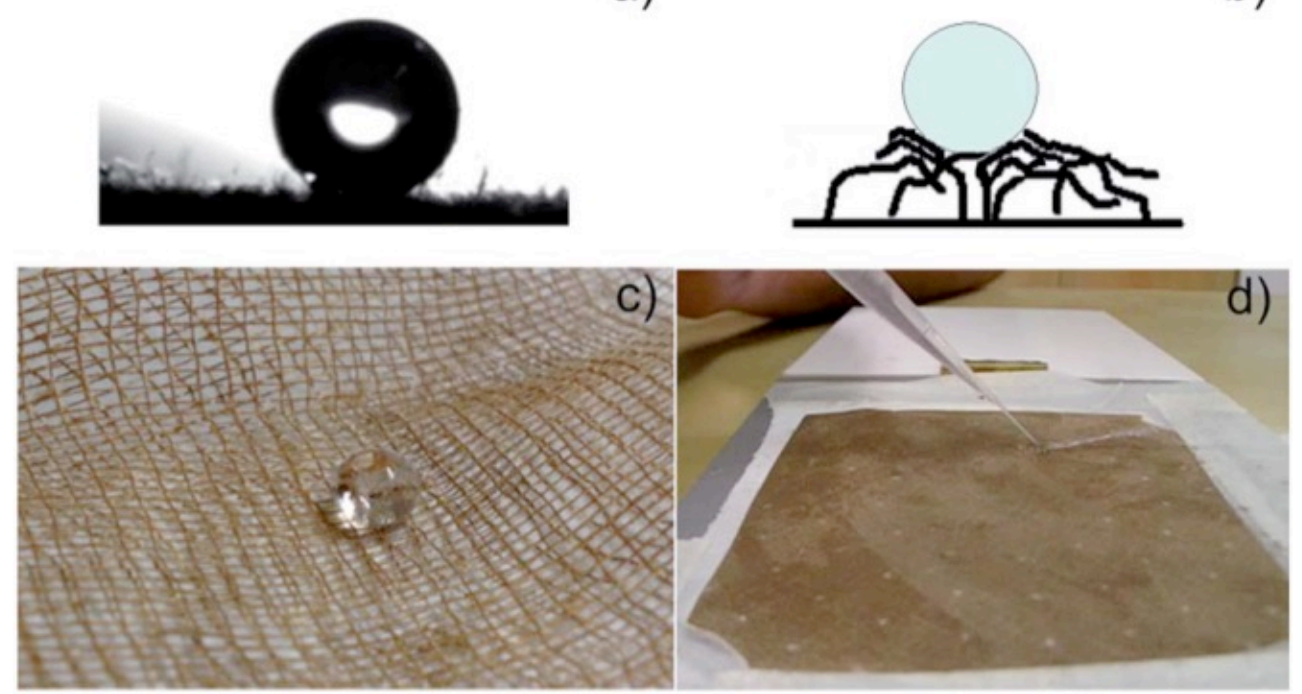

Fig. 7. Wetting behavior of water on Diamond Like Carbon (DLC)-coated cotton. (a) An optical image of static water droplets $(3 \mathrm{~mL})$ at a $\mathrm{CA}$ of $170^{\circ}$ on the respective DLC-coated textiles modified by a 60 min plasma pretreatment. (b) Schematic of the elastic deformation of the protruding cotton fibres due to bundles formed at the water-air interface. (c) Plasma-treated and DLC-coated gauze showing superhydrophobic properties on a mesh with very large pore size. (d) Optical image demonstrating that a jet of water is deflected by the DLC coated cotton fabrics demonstrating robust superhydrophobic properties. 




Fig. 8. FE-SEM images of meshes at different magnification: Top) the as-received copper mesh surface. Bottom)

The mesh membrane coated with Ag. 


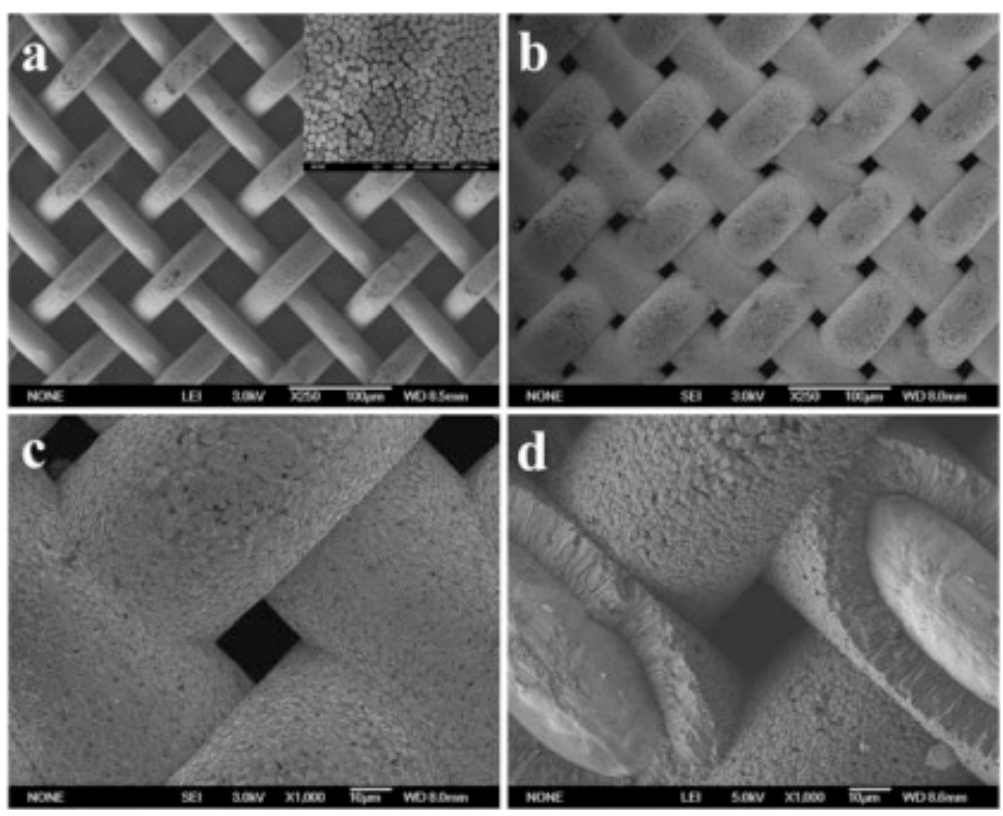

Fig. 9. SEM images of the underlying mesh and zeolite coated mesh membrane (a) Large scale view of the bare stainless steel mesh with an average pore diameter of about $42 \mathrm{~mm}$, the inset is a magnified image of the mesh after the seeding process. (b) $\mathrm{Al}$ arge scale image of the ZCMF-12 coated mesh membrane. (c) Magnified view of a single pore, and its (d) cross-sectional view. 